\title{
Exploiting Large-Scale Correlations to Detect Continuous Gravitational Waves
}

\author{
Holger J. Pletsch ${ }^{1, *}$ and Bruce Allen ${ }^{1,2}$, \\ ${ }^{1}$ Max-Planck-Institut für Gravitationsphysik (Albert-Einstein-Institut), Callinstraße 38, D-30167 Hannover, Germany \\ ${ }^{2}$ Department of Physics, University of Wisconsin - Milwaukee, P.O. Box 413, Wisconsin, 53201, USA
}

\begin{abstract}
Fully coherent searches (over realistic ranges of parameter space and year-long observation times) for unknown sources of continuous gravitational waves are computationally prohibitive. Less expensive hierarchical searches divide the data into shorter segments which are analyzed coherently, then detection statistics from different segments are combined incoherently. The novel method presented here solves the long-standing problem of how best to do the incoherent combination. The optimal solution exploits largescale parameter-space correlations in the coherent detection statistic. Application to simulated data shows dramatic sensitivity improvements compared with previously available (ad hoc) methods, increasing the spatial volume probed by more than 2 orders of magnitude at lower computational cost.
\end{abstract}

PACS numbers: 04.80.Nn, 95.55.Ym, 95.75.-z, 97.60.Jd

Searching for CW Sources. - Direct detection of gravitational waves is the most significant remaining test of Einstein's General Theory of Relativity, and will become an important new astronomical tool.

Rapidly rotating neutron stars are expected to generate continuous gravitational-wave $(\mathrm{CW})$ signals via various mechanisms [1, 2, 3, 4, 5]. Most such stars are electromagnetically invisible, but might be detected and studied via gravitational waves. Recent simulations of neutron star populations [6, 7] suggest that CW sources might eventually be detected with new instruments such as LIGO [8, 9]. World-wide efforts are underway to search for $\mathrm{CW}$ signals [10, 11, 12] and observational upper limits already place some constraints on neutron star physics [13, 14].

Because the expected CW signals are weak, sensitive data analysis methods are needed to extract these signals from detector noise. A powerful method is derived in Ref. [15]. This scheme is based on the principle of maximum likelihood detection, which leads to coherent matched filtering. Rotating neutron stars emit monochromatic $\mathrm{CW}$ signals, apart from a slowly changing intrinsic frequency. But the terrestrial detector location Dopplermodulates the amplitude and phase of the waveform, as the Earth moves relative to the solar system barycenter (SSB). The parameters describing the signal's amplitude variation may be analytically eliminated by maximizing the coherent matched-filtering statistic [15]. The remaining search parameters describing the signal's phase are the source's sky location, frequency and frequency derivatives. The resulting coherent detection statistic is called the $\mathcal{F}$-statistic.

This work considers isolated CW sources whose frequency varies linearly with time in the SSB frame. The corresponding phase parameter-space $\mathcal{P}$ is fourdimensional. Standard "physical" coordinates on $\mathcal{P}$ are the frequency $f\left(t_{0}\right)$ at some fiducial time $t_{0}$, the frequency's first time derivative $\dot{f}$, and a unit vector $\mathbf{n}=$ $(\cos \delta \cos \alpha, \cos \delta \sin \alpha, \sin \delta)$ on the two-sphere $S^{2}$, pointing from the SSB to the source. Here $\alpha$ and $\delta$ are right ascension and declination. Thus, a point in parameter space $\mathrm{p} \in \mathcal{P}$ may be labeled by $\mathrm{p}=\left\{f\left(t_{0}\right), \dot{f}, \mathbf{n}\right\}$. The $\mathcal{F}$-statistic $\mathcal{F}_{\mathrm{p}}[h]$ is a functional of the detector data set $h$, and is a function of the point in parameter space $\mathrm{p} \in \mathcal{P}$.

All-sky searches for unknown CW sources using the $\mathcal{F}$-statistic are computationally expensive. For maximum sensitivity, one must convolve the full data set with signal waveforms (templates) corresponding to all possible sources. But the number of templates required for a fully coherent search increases as a high power of the observation time. For one year of data, the computational cost to search a realistic range of parameter space exceeds the total computing power on Earth [15, 16]. Thus a fully coherent search is limited to much shorter observation times.

Searching year-long data sets is accomplished by less costly hierarchical semicoherent methods [17, 18, 19]. The data is broken into segments of duration $T$, where $T$ is much smaller than one year. Each segment is analyzed coherently, computing the $\mathcal{F}$-statistic on a coarse grid of templates. Then the $\mathcal{F}$ values from all segments (or statistics derived from $\mathcal{F}$ ) are incoherently combined using a common fine grid of templates, discarding phase information between segments. Among the current semicoherent strategies, the Stack-Slide method [17, 18] sums $\mathcal{F}$ values along putative signal tracks in the time-frequency plane. The Hough transform method [19] sums $H\left(\mathcal{F}-\mathcal{F}_{\text {th }}\right)$ where $\mathcal{F}_{\text {th }}$ is a constant predefined threshold. The Heavyside function $H(\mathrm{x})$ is unity for positive $\mathrm{x}$ and vanishes elsewhere. This latter technique is currently used by Einstein@Home [12], a public distributed computing project carrying out the most sensitive all-sky CW searches.

A central long-standing problem in these semicoherent methods is the design of, and link between, the coarse and fine grids. Current methods, while creative and clever, are arbitrary and ad hoc constructions. This work removes all arbitrariness by finding the optimal solution for the incoherent step. The key quantity is the fractional loss, called mismatch $\mathcal{M}$, in expected $\mathcal{F}$-statistic (or sum of $\mathcal{F}$-statistics in the incoherent step) for a given signal $\mathrm{p}$ at a nearby grid point $\mathrm{p}^{\prime}$. Locally Taylor-expanding $\mathcal{M}$ (to quadratic order) in the differences of the coordinates 
$\left\{f\left(t_{0}\right), \dot{f}, \mathbf{n}\right\}$ of $\mathrm{p}$ and $\mathrm{p}^{\prime}$ defines a signature ++++ metric $\mathrm{d} s^{2}[16,20,21,22]$. Current methods consider parameter correlations in $\mathcal{F}$ to only linear order in $T$.

The $\mathcal{F}$-statistic has large-scale correlations [23, 24] in the physical coordinates $\left\{f\left(t_{0}\right), \dot{f}, \mathbf{n}\right\}$, extending outside a region in which the mismatch is well-approximated by the metric given above [25]. Recent work [24] has shown that (for a given signal) the region where the expected $\mathcal{F}$ statistic has maximal value may be described by a separate equation for each order of $T$, when $T$ is small compared to one year. The solutions to these equations are hypersurfaces in $\mathcal{P}$, whose intersections are the extrema of (an approximation to) $\mathcal{F}$.

For currently used values of $T$ (a day or longer) it is also crucial to consider the fractional loss of $\mathcal{F}$ to second order in $T$ [24]. For source frequencies $\gtrsim 1 \mathrm{kHz}$ and for values of $T \gtrsim 60 \mathrm{~h}$, additional orders in $T$ would be needed.

The new method. - This work exploits the large-scale correlations in the coherent detection statistic $\mathcal{F}$ to construct a significantly improved semicoherent search technique for CW signals. The novel method is optimal if the semicoherent detection statistic is taken to be the sum of one coarse-grid $\mathcal{F}$-statistic value from each data segment, and makes four important improvements.

First, the improved understanding of large-scale correlations yields new coordinates on $\mathcal{P}$. The metric in these coordinates accurately approximates the mismatch in $\mathcal{F}$ [25] in each segment. Hence, the optimal (closest) coarse-grid point from each segment can be determined for any given fine-grid point in the incoherent combination step.

Second, in the new coordinates we find the first analytical metric for the semicoherent detection statistic to construct the optimal fine grid (minimum possible number of points). Previous ad hoc approaches obtain the ne grid by rening the coarse grid in three dimensions, $\dot{f}$ and $\mathbf{n}$. Here, the explicit incoherent-step metric shows that refinement is only needed in one dimension, $\dot{f}$. This greatly reduces the computational cost at equal detection sensitivity, although it also reduces the accuracy of the estimated source parameters. But this is a very profitable trade, because in a hierarchical search the primary goal of the first stages is to discard the uninteresting regions of parameter space. Later follow-up stages use longer coherent integrations to more accurately determine the source parameters.

Third, existing techniques combine the coherent results less effectively than our method, because they do not use metric information beyond linear order in $T$. This leads to a higher sensitivity at equal computational cost.

Fourth, the new technique can simultaneously do a Stack-Slide-like summing of $\mathcal{F}$ values and a Hough-like summing of $H\left(\mathcal{F}-\mathcal{F}_{\text {th }}\right)$, with a lower total computational cost than either one of these methods individually.

For a given $\mathrm{CW}$ source with realistic phase parameter values $(f \lesssim 1 \mathrm{kHz},|\dot{f}| \lesssim f / 50 \mathrm{yr})$ and coherent data segment lengths $T \lesssim 60 \mathrm{~h}$, the large-scale correlations of the
$\mathcal{F}$-statistic are well described by the first- and second-order (in $T$ ) equations [24]:

$$
\begin{aligned}
& \nu(t)=f(t)+f(t) \dot{\boldsymbol{\xi}}(t) \cdot \mathbf{n}+\dot{f} \boldsymbol{\xi}(t) \cdot \mathbf{n}, \\
& \dot{\nu}(t)=\dot{f}+f(t) \ddot{\boldsymbol{\xi}}(t) \cdot \mathbf{n}+2 \dot{f} \dot{\boldsymbol{\xi}}(t) \cdot \mathbf{n}, \\
& \text { where } f(t) \equiv f\left(t_{0}\right)+\left(t-t_{0}\right) \dot{f} .
\end{aligned}
$$

Here $\boldsymbol{\xi}(t) \equiv \mathbf{r}_{\text {orb }}(t) / c$, with $\mathbf{r}_{\text {orb }}(t)$ denoting the vector from the Earth's barycenter to the SSB, and $c$ the speed of light. The quantities $\nu(t)$ and $\dot{\nu}(t)$ can be interpreted as the source's instantaneous frequency and frequency derivative at the Earth's barycenter at time $t$.

The parameters $\nu$ and $\dot{\nu}$ provide new coordinates on $\mathcal{P}$. It is also useful to introduce new (real-valued) sky coordinates $n_{\mathrm{x}}$ and $n_{\mathrm{y}}$ (as in [26]):

$$
n_{\mathrm{x}}(t)+i n_{\mathrm{y}}(t)=f(t) \tau_{E} \cos \delta_{D} \cos \delta \mathrm{e}^{i\left[\alpha-\alpha_{D}(t)\right]} .
$$

Here $\tau_{E}=R_{E} / c \approx 21 \mathrm{~ms}$ is the light travel time from the Earth's center to the detector, and $\alpha_{D}(t), \delta_{D}$ are the detector position at time $t$. The metric separation $\mathrm{d} s^{2}$ is

$$
\begin{aligned}
\mathrm{d} s^{2} / \pi^{2}= & \mathrm{d} \nu^{2} T^{2} / 3+\gamma^{2} \mathrm{~d} \dot{\nu}^{2} T^{4} / 180+2 \mathrm{~d} n_{\mathrm{x}}^{2}+2 \mathrm{~d} n_{\mathrm{y}}^{2} \\
& -4 \mathrm{~d} \nu \mathrm{d} n_{\mathrm{y}} T /(\pi \ell)+4 \mathrm{~d} \dot{\nu} \mathrm{d} n_{\mathrm{x}} T^{2} /(\pi \ell)^{2} .
\end{aligned}
$$

In defining differences in coordinates $\left\{\nu, \dot{\nu}, n_{\mathrm{x}}, n_{\mathrm{y}}\right\}$, the time $t$ in Eqs. (1) and (2) is the midpoint of the data segment spanning times $[t-T / 2, t+T / 2]$, and $\gamma=1$. To simplify the form of the metric, $T$ is taken to be a positive integer number $\ell$ of sidereal days.

The new coordinates $\left\{\nu, \dot{\nu}, n_{\mathrm{x}}, n_{\mathrm{y}}\right\}$ have important advantages over the original coordinates $\{f, \dot{f}, \mathbf{n}\}$. The metric is explicitly coordinate-independent (showing that $\mathcal{P}$ is flat). In fact, the region around a point $\mathrm{p}$ in which the mismatch $\mathcal{M}$ is well-approximated by $\mathrm{d} s^{2}$ is much larger [25].

Consider a segment of data $h_{\mathrm{p}}$ which contains a strong $\mathrm{CW}$ source with phase parameters $\mathrm{p}$. If the sky separation patch is small enough to neglect the $\mathrm{d} n_{\mathrm{x}}$ and $\mathrm{d} n_{\mathrm{y}}$ terms in Eq. (3), then $\mathcal{F}_{\mathrm{p}^{\prime}}\left[h_{\mathrm{p}}\right]$ is extremized for all $\mathrm{p}^{\prime}$ that have the same values of $\nu$ and $\dot{\nu}$ as p. This set of points in $\mathcal{P}$ forms a two-dimensional surface $\mathrm{d} \nu=\mathrm{d} \dot{\nu}=0$. Thus, for all sources within the sky patch, there exists a different $(f, \dot{f})$ pair with those same values of $\nu$ and $\dot{\nu}$. This property is exploited by the new method.

An implementation. - The data set is divided into $N$ segments of length $T$ (potentially including short gaps in operation) labeled by the integer $j=1, \ldots, N$. The segments span time intervals $\left[t_{j}-T / 2, t_{j}+T / 2\right]$. The detector-time midpoint of segment $j$ is $t_{j}$ and $t_{0} \equiv$ $\frac{1}{N} \sum_{j=1}^{N} t_{j}$ is the fiducial time.

Every segment is analyzed coherently on a coarse grid in $\mathcal{P}$. This grid is constructed so that no point in $\mathcal{P}$ is farther than a specified distance from some coarse-grid point, where the distance measure is defined by the metric of Eq. (3). To simplify the grid construction, large frequency bands are analyzed by breaking them into many 
narrow sub-bands. For each segment $j$, and at each coarse grid point, the $\mathcal{F}$-statistic is evaluated, and "stats" are obtained. Here, the word "stat" denotes the two-tuple $\left(\mathcal{F}_{j}, H\left(\mathcal{F}_{j}-\mathcal{F}_{\text {th }}\right)\right)$.

For simplicity, the same coarse grid is used for all data segments: the Cartesian product of a rectangular grid in $f, \dot{f}$ and a grid on the sky-sphere $\mathbf{n} \in S^{2}$. The spacings are $\Delta f=\sqrt{12 m} /(\pi T)$ and $\Delta \dot{f}=\sqrt{720 m} /\left(\pi T^{2}\right)$, where $m$ is the one-dimensional metric mismatch parameter [11]. The spacing of the coarse sky grid is chosen so that the $\mathrm{d} n_{\mathrm{x}}$ and $\mathrm{d} n_{\mathrm{y}}$ terms in Eq. (3) may be neglected. When orthogonally projected onto the equatorial unit disk, the sky grid is uniform, and contains $\approx 2 \pi /(\Delta \varphi)^{2}$ points, with $\Delta \varphi=\sqrt{2 m} /\left(\pi f \tau_{E} \cos \delta_{D}\right)$.

The incoherent step combines the "stats" obtained by the coherent analysis, using a fine grid in $\mathcal{P}$. At each point in the fine grid, a "stat" value is obtained by summing one stat value from each of the $N$ coarse grids. The coarse grid point is the one with the same sky position as the fine grid point, which has the smallest metric separation from Eq. (3). The final result is a "stat" value at each point on the fine grid. The first element of the stat is the sum of the $\mathcal{F}$-statistic values from the coarse-grid points. The second element is a number count, reflecting the number of data segments in which $\mathcal{F}_{\text {th }}$ was exceeded. A detectable CW signal leads to a fine-grid point with a high number count and a large sum of $\mathcal{F}$-statistics.

The spacing of the fine grid is determined from the metric for the fractional loss of the expected $\sum_{j=1}^{N} \mathcal{F}_{j}$ due to parameter offsets between a putative signal location and a fine grid point. This may be calculated as proposed in [17], by averaging the coarse-grid metric over the $N$ different segments. Since each coarse-grid metric is no longer calculated at the data-segment midpoints (but at $t_{0}$ ), the coefficients change between segments because of the timedependence of the parameter-space coordinates. For our choices of $t_{0}$ and $T$, the only additional term in the metric Eq. (3) that does not average to zero is $\left(t_{j}-t_{0}\right)^{2} T^{2} \mathrm{~d} \dot{\nu}^{2} / 3$. The averaged metric is the diagonal part of Eq. (3) with $\gamma^{2}=1+60 \sum_{j=1}^{N}\left(t_{j}-t_{0}\right)^{2} /\left(N T^{2}\right)$, where the coordinate offsets are calculated at the fiducial time $t_{0}$. Thus, the fine grid may be identical to the coarse grid except that the spacing $\Delta \dot{f}$ is smaller by a factor $\gamma$. This is of order $N$ when the number of data segments is large. No further refinement in frequency or sky position is needed. Coherent integration over the total observation time would require refining both $\Delta \dot{f}$ and $\Delta f$ (increasing points $\propto N^{3}$ ), plus similar sky refinements.

Performance comparison. - Monte Carlo simulations are used to illustrate the improved performance of this method compared with the conventional Hough transform technique [19]. The software tools used are part of LALApps [27] and employ accurate barycentering routines with timing errors below $3 \mu \mathrm{s}$. To provide a realistic comparison, simulated data covered the same time intervals as the input data used for the current (S5R5) Einstein@Home search [12]. Those data, from LIGO Hanford (H1, 4km) and LIGO Livingston (L1, 4km), are not contiguous, but contain gaps when the detectors are not operating. The total time interval spanned is about 264 days, containing 121 data segments of duration $25 \mathrm{~h}$ (so approximately $\ell=1$ ).

The false alarm probabilities are obtained using 5000 simulated data sets with different realizations of stationary Gaussian white noise, with one-sided strain spectral density $\sqrt{S_{h}}=3.25 \times 10^{-22} \mathrm{~Hz}^{-1 / 2}$. To find the detection probabilities, different $\mathrm{CW}$ signals with fixed strain amplitude $h_{0}$ are added. The parameters [15] are randomly drawn from uniform distributions in $\cos ($ inclination $\iota$ ), polarization $\psi$, initial phase $\phi_{0}$, the entire sky, $f\left(t_{0}\right) \in$ $[100.1,100.3] \mathrm{Hz}$, and $\dot{f} \in[-1.29,-0.711] \mathrm{nHz} / \mathrm{s}$.

Figure 1 compares the performance of the different methods. The receiver operating characteristic is the detection probability as a function of false alarm probability, at fixed source strain amplitude $h_{0}=6 \times 10^{-24}$. Because the number count (using $\mathcal{F}_{\mathrm{th}}=2.6$ ) is discrete, the two "curves" in Fig. 1 consist of discrete points. Our method (using either number counts or summed $\mathcal{F}$ as a detection statistic) is superior to the conventional Hough technique.

In addition, this method is computationally faster. The comparison used identical coherent stages ( $m=0.3$, with 2981 coarse-grid points) for both this method and the conventional Hough. But using different fine grids in the incoherent step, this method's fine grid had 506 times as many points as the coarse grid, but the Hough fine grid had 7056 times as many points. In spite of using 14 times fewer finegrid points, our method has substantially higher sensitivity.

Figure 2 compares the methods. It shows the detection efficiencies for different values of source strain amplitude $h_{0}$, at a fixed $1 \%$ false alarm probability. As above, each point in Fig. 2 is obtained by analyzing 2000 simulated data sets. Again, this technique in both modes of operation performs substantially better than the Hough

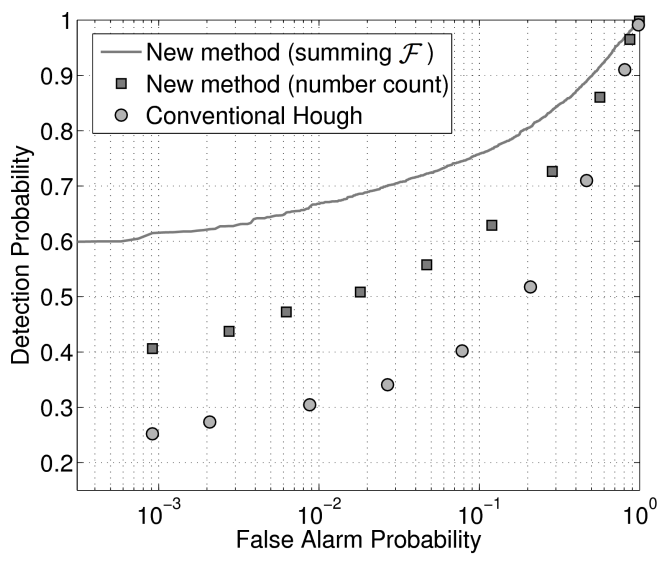

FIG. 1: Receiver operating characteristic curve. The new method performs better than the conventional Hough technique. 


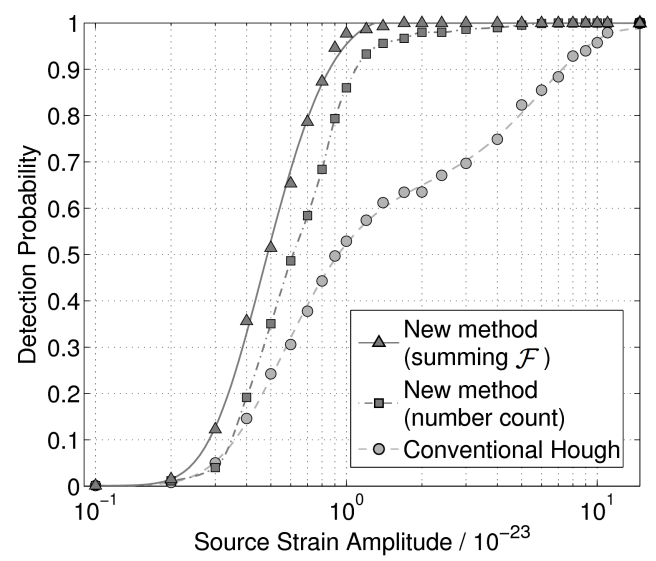

FIG. 2: Detection probability as a function of source strain amplitude $h_{0}$, at $1 \%$ false alarm probability.

method. For example, the $h_{0}$ value needed to obtain $90 \%$ detection probability with our technique in number-count operation mode is smaller by a factor $\approx 6$ than the $h_{0}$ needed by the Hough method: the "distance reach" [15] of our technique is $\approx 6$ times larger. This increases the number of potentially detectable sources by more than 2 orders of magnitude, since the "visible" spatial volume increases as the cube of the distance. The lower computational cost of our method would also allow increases in $N$ or $T$, even further improving the sensitivity.

These results are qualitatively independent of frequency, as confirmed in additional comparisons.

Conclusions. - A novel semicoherent technique for detecting $\mathrm{CW}$ sources has been described. In contrast to previous approaches, the new method exploits largescale parameter-space correlations in the coherent detection statistic $\mathcal{F}$ to optimally solve the subsequent incoherent combination step. For coherent integration times $T \lesssim 60 \mathrm{~h}$, the correlations are well-described by the second-order (in $T$ ) formulae presented here. The method should be extendible to longer coherent integration times by including higher orders. It could be extended to search for CW sources in binary systems, as well as to spacebased detectors. The method also has applicability in radio, $\mathrm{X}$ - and $\gamma$-ray astronomy (searches for weak radio or $\gamma$-ray pulsars, or pulsations from X-ray binaries).

Realistic Monte Carlo simulations show that our technique is much more sensitive than the conventional Hough method (current most sensitive all-sky CW search technique). The technique presented here is also computationally simpler, and more efficient.

The LIGO Scientific Collaboration is currently working to deploy this technique on the Einstein@Home project [12], starting with LIGO S6 data. The combination of a better search technique, and more sensitive data, greatly increases the chance of making the first gravita- tional wave detection of a $\mathrm{CW}$ source. The detection of $\mathrm{CW}$ signals will provide new means to discover and locate neutron stars, and will eventually provide unique insights into the nature of matter at high densities.

We thank R. Prix, M. A. Papa, C. Messenger, B. Krishnan and B. Knispel for helpful discussions. This work was supported by the Max Planck Gesellschaft and by U.S. National Science Foundation Grant No. 0555655 and No. 0701817.

* Electronic address: Holger.Pletsch@ aei.mpg.de

$\dagger$ Electronic address: Bruce.Allen@aei.mpg.de

[1] B. J. Owen et al., Phys. Rev. D 58, 084020 (1998).

[2] G. Ushomirsky, C. Cutler, and L. Bildsten, Mon. Not. Roy. Astron. Soc. 319, 902 (2000).

[3] C. Cutler, Phys. Rev. D 66, 084025 (2002).

[4] D. I. Jones and N. Andersson, Mon. Not. Roy. Astron. Soc. 331, 203 (2002).

[5] B. J. Owen, Phys. Rev. Lett. 95, 211101 (2005).

[6] B. Knispel and B. Allen, Phys. Rev. D 78, 044031 (2008).

[7] C. J. Horowitz and K. Kadau, Phys. Rev. Lett. 102, 191102 (2009).

[8] A. Abramovici et al., Science 256, 325 (1992).

[9] B. C. Barish and R. Weiss, Physics Today 52, 44 (1999).

[10] B. Abbott et al. (The LIGO Scientific Collaboration), Phys. Rev. D 77, 022001 (2008).

[11] B. Abbott et al. (The LIGO Scientific Collaboration), Phys. Rev. D 79, 022001 (2009).

[12] Einstein@Home: http://einstein.phys.uwm.edu/.

[13] B. Abbott et al. (The LIGO Scientific Collaboration), Astrophys. J. Lett. 683, L45 (2008).

[14] B. Abbott et al. (The LIGO Scientific Collaboration), Phys. Rev. Lett. 102, 111102 (2009).

[15] P. Jaranowski, A. Królak, and B. F. Schutz, Phys. Rev. D 58, 063001 (1998).

[16] P. R. Brady et al., Phys. Rev. D 57, 2101 (1998).

[17] P. R. Brady and T. Creighton, Phys. Rev. D 61, 082001 (2000).

[18] C. Cutler, I. Gholami, and B. Krishnan, Phys. Rev. D 72, 042004 (2005).

[19] B. Krishnan et al., Phys. Rev. D 70, 082001 (2004).

[20] R. Balasubramanian, B. S. Sathyaprakash, and S. V. Dhurandhar, Phys. Rev. D 53, 3033 (1996).

[21] B. J. Owen, Phys. Rev. D 53, 6749 (1996).

[22] R. Prix, Phys. Rev. D 75, 023004 (2007).

[23] R. Prix and Y. Itoh, Class. Quant. Grav. 22, S1003 (2005).

[24] H. J. Pletsch, Phys. Rev. D 78, 102005 (2008).

[25] Using $\left\{\nu, \dot{\nu}, n_{\mathrm{x}}, n_{\mathrm{y}}\right\}$ coordinates the metric $\mathrm{d} s^{2}$ remains accurate up to $\mathcal{M}=0.3$. In contrast, in the $\{f, \dot{f}, \mathbf{n}\}$ coordinates $\mathrm{d} s^{2}$ can yield errors greater than $10 \%$ for mismatches as small as 0.001 . In part, this is because the metric in $\{f, \dot{f}, \mathbf{n}\}$ varies significantly over the $\mathcal{M}=0.3$ region.

[26] P. Astone et al., Phys. Rev. D 65, 042003 (2002).

[27] http://www.lsc-group.phys.uwm.edu/daswg/. 